\title{
Capacitation-associated protein tyrosine phosphorylation and membrane fluidity changes are impaired in the spermatozoa of asthenozoospermic patients
}

\author{
Mariano G Buffone, Juan C Calamera, Sandra V Verstraeten ${ }^{1}$ and Gustavo F Doncel ${ }^{2}$ \\ Laboratorio de Estudios en Reproducción (LER), Buenos Aires, Argentina, 'Departamento de Química Biológica, \\ IQUIFIB (CONICET-UBA), Facultad de Farmacia y Bioquímica, Universidad de Buenos Aires, Buenos Aires, \\ Argentina and ${ }^{2}$ CONRAD, Department of Obstetrics and Gynecology, The Jones Institute for Reproductive \\ Medicine, Eastern Virginia Medical School, 601 Colley Ave., Norfolk, Virginia 23507, USA
}

Correspondence should be addressed to G F Doncel; Email: DoncelGF@evms.edu

\begin{abstract}
Sperm protein tyrosine phosphorylation has been associated with capacitation, motility changes, zona binding, and fertilizing ability. We previously demonstrated that gradient-isolated human sperm subpopulations differ in their plasma membrane composition, their ability to phosphorylate proteins in tyrosine residues, and their capacity to undergo hyperactivation. In this study, we have characterized capacitation-associated changes in protein tyrosine phosphorylation and membrane fluidity in spermatozoa of asthenozoospermic and normozoospermic patients consulting for infertility. Semen samples were studied at baseline and after a capacitating incubation with or without the addition of a permeable cAMP analog and a phosphodiesterase inhibitor. Basic sperm and computer-assisted motion parameters, hyperactivation, protein tyrosine phosphorylation (immunofluorescence and Western blot), and membrane fluidity (fluorescent Laurdan probe) were the main study parameters. In comparison with normozoospermic and proven-fertile donor semen, asthenozoospermic samples showed lower motility, velocity, and amplitude of lateral head displacement, both originally and after a 6-h capacitating incubation. Unlike those in normal samples, asthenozoospermic spermatozoa were unable to increase protein tyrosine phosphorylation during capacitation. Such impairment, however, was overcome when they were incubated with a membrane-permeable cAMP analog and a phosphodiesterase inhibitor, indicating a possible membrane defect. Confirming this hypothesis, plasma membranes of asthenozoospermic sperm showed decreased fluidity (increased Laurdan polarization), even after a capacitating incubation.

In conclusion, spermatozoa from functional asthenozoospermic samples may owe their poor motility, and their inability to properly capacitate and develop hyperactivation, to an impairment in the tyrosine phosphorylation of critical proteins caused by decreased membrane fluidity. These findings suggest a molecular pathogenetic mechanism for a common seminal pathology associated with male infertility.

Reproduction (2005) 129 697-705
\end{abstract}

\section{Introduction}

Capacitation-associated protein tyrosine phosphorylation has been described in the spermatozoa of numerous species, including humans (Visconti et al. 1995a, Carrera et al. 1996, Pukazhenthi et al. 1998, Si \& Okuno 1999, Yeung et al. 1999). Furthermore, it has been associated with acquisition of the fertilizing ability of sperm (Visconti et al. 1995a, Sakkas et al. 2003) and development of sperm hyperactivation (Nassar et al. 1999, Si \& Okuno 1999, Yunes et al. 2003).

Tyrosine phosphorylation in spermatozoa is regulated by a complex series of molecular events involving membrane cholesterol efflux and hyperpolarization, increase in intracellular $\mathrm{NaHCO}_{3}, \mathrm{Ca}^{2+}$ and $\mathrm{CAMP}$, and activation of phosphokinases (Visconti \& Kopf 1998, Visconti et al. 2002). Following capacitation-associated cholesterol efflux and its consequent increase in membrane fluidity, sperm show a rise in cAMP produced by a $\mathrm{NaHCO}_{3}$ dependent soluble adenyl cyclase bound to the cytoskeletal fraction in mature sperm, which precedes an upregulation of sperm motion parameters, hypermotility, and tyrosine phosphorylation (Visconti et al. 1995b, Leclerc et al. 1996, Bajpai \& Doncel 2003, Luconi et al. 2005). Tyrosine (protein tyrosine kinases; PTK) and cAMPdependent (protein kinase A; PKA) kinases are involved in these processes as their inhibition in human spermatozoa decreases tyrosine phosphorylation and motility concomitantly (Bajpai \& Doncel 2003, Bajpai et al. 2003). 
Studying sperm subpopulations from normozoospermic human semen samples, we also demonstrated that Percoll-isolated low-quality spermatozoa showed an impairment in the development of tyrosine phosphorylation during capacitation (Buffone et al. 2004). Such deficiency, however, was overcome with permeable activators of PKA, which led us to postulate that the main defect in those spermatozoa was localized upstream of PKA, possibly at the plasma membrane. Changes in plasma membrane composition have been associated with both capacitation (Davis 1981, Cross 1998) and sperm pathology (Calamera et al. 2003). Considering these findings as well as preliminary data gathered from samples with abnormal motility, we hypothesized that such combined alteration may be the underlying cause of certain sperm pathologies, especially those displaying low motility (Yunes et al. 2003).

In order to verify this hypothesis and to expand the characterization of such molecular defects in pathological sperm, we have studied the capacitation-associated changes of protein tyrosine phosphorylation and membrane fluidity in spermatozoa of asthenozoospermic, normozoospermic, and proven-fertile human subjects. Herein, we describe the results of this investigation.

\section{Materials and Methods}

\section{Semen collection and analysis}

Semen samples were obtained by masturbation from patients consulting our center for infertility. After a basic semen analysis, the unused parts of these samples were included in the study with the patients' consent. Subjects $(n=34)$ were 25-40 years of age and collected semen after 3-5 days of sexual abstinence. Ejaculates were allowed to liquify for $1 \mathrm{~h}$ at room temperature and sperm concentration and motility were assessed using a computer-assisted semen analysis (Hamilton Thorne IVOS V10.8s; Hamilton Thorne Research, Danvers, MA, USA). Sperm viability was assessed by light microscopy in the original semen samples as well as the isolated fractions at all incubation times using the Eosin Y assay (World Health Organization 1999). Sperm morphology was assessed by Kruger's strict criteria (Kruger et al. 1986).

Sperm samples were classified as normozoospermic (sperm concentration $>20 \times 10^{6}$ spermatozoa/ml, percentage of motile cells $>50 \%$, percentage of viable spermatozoa $\geq 80 \%$, and percentage of normal forms $\geq 14 \%$ ) or asthenozoospermic (sperm concentration $>20 \times 10^{6}$ spermatozoa/ml, percentage of motile cells $<40 \%$, percentage of viable spermatozoa $\geq 80 \%$, and percentage of normal forms $\geq 14 \%$ ). We also studied samples from seven sperm donors of proven fertility.

\section{Motility parameters and sperm hyperactivation}

Aliquots of each sperm suspension were loaded into a $20 \mu \mathrm{m}$ deep disposable chamber (Microcell; Conception
Technologies, San Diego, CA, USA) pre-warmed at $37^{\circ} \mathrm{C}$. Computer-assisted sperm motion analysis was performed using a Hamilton Thorne digital image analyzer (HTRIVOS v $10.8 \mathrm{~s}$; Hamilton Thorne Research) before and after a $6 \mathrm{~h}$ capacitating incubation. At least 300 spermatozoa and five fields were assessed.

Eight motion parameters were assessed in this study: (1) motility (\%); (2) average path velocity (VAP, $\mu \mathrm{m} / \mathrm{s})$; (3) track speed or curvilinear velocity $(\mathrm{VCL}, \mu \mathrm{m} / \mathrm{s})$; (4) progressive or straight-line velocity (VSL, $\mu \mathrm{m} / \mathrm{s}) ;(5)$ straightness (STR, \%); (6) beat cross frequency (BCF, Hz); (7) linearity (LIN, \%); (8) lateral head amplitude (ALH, $\mu \mathrm{m})$. The settings used during the analysis were: frames acquired, 30; frame rate, $60 \mathrm{~Hz}$; minimum contrast, 85; minimum cell size, 4 pixels; straightness threshold, 80\%; low VAP cut off, $5 \mu \mathrm{m} / \mathrm{s}$; medium VAP cut off, $25 \mu \mathrm{m} / \mathrm{s}$; head size - non-motile, 12 pixels; head intensity - nonmotile, 130 Units $(U)$; static head size, 0.68-2.57 pixels; static head intensity, $0.31-1.21 \mathrm{U}$; static elongation, $23-100 \%$. The playback function was used to accurately identify motile cells. Hyperactivated motility (\%) was defined as motility with starspin or high-amplitude trashing patterns and short trajectory distances (Burkman 1984). This percentage represents the portion of motile spermatozoa displaying hyperactivated movement. The criteria for detecting hyperactivated spermatozoa were: VCL $>150 \mu \mathrm{m} / \mathrm{s} ; \mathrm{ALH}>7.0 \mu \mathrm{m} ;$ LIN $<50 \%$ (Mortimer et al. 1998).

\section{Preparation of spermatozoa}

Sperm cells were separated from seminal plasma by dilution with Ham's F10 medium (Gibco BRL, Grand Island, NY, USA) containing $3 \mathrm{mg} / \mathrm{ml}$ bovine serum albumin (BSA; Sigma, St Louis, MO, USA) (Ham/BSA) and centrifugation at $400 \mathrm{~g}$ for $5 \mathrm{~min}$ (three times). The pellet was resuspended in $1 \mathrm{ml} \mathrm{Ham} / \mathrm{BSA}$ and an aliquot of it was used to assay sperm concentration and motility. This was considered the first incubation time (T0). Sperm concentration was adjusted to approximately $10 \times 10^{6}$ spermatozoa/ml and incubated for $6 \mathrm{~h}$ (T6) at $37^{\circ} \mathrm{C}$ in $5 \%$ $\mathrm{CO}_{2}$. In some instances, sperm were separated from seminal plasma using Percoll-gradient centrifugation (500 $\mathrm{g}$ for $20 \mathrm{~min}$ ) and subsequent washing with Ham/BSA. To increase intracellular levels of cyclic AMP (cAMP), sperm where incubated for $1 \mathrm{~h}$ with dibutyryl cAMP (dbcAMP; $1 \mathrm{mM})$ and pentoxifylline (PTX; $1 \mathrm{mM})$.

\section{Indirect immunofluorescence of spermatozoa}

Immunofluorescence was employed to examine the subcellular localization of proteins phosphorylated in tyrosine residues as well as the incidence of tyrosine-phosphorylated sperm in a given population. Spermatozoa from the different groups were capacitated during various periods of time and washed twice with phosphate-buffered saline (PBS). Sperm concentration was adjusted to $5 \times 10^{6}$ cells/ml and $15 \mu \mathrm{l}$ of the sperm suspension was spotted 
onto eight-well glass slides. Cells were air-dried on the slides, fixed, and permeabilized with methanol for $30 \mathrm{~min}$ at room temperature. The slides were incubated with anti-phosphotyrosine antibody PY20 (ICN Biomedicals Inc., Aurora, OH, USA), diluted 1:20 $(50 \mu \mathrm{g} / \mathrm{ml})$ in PBS- $-1 \%$ BSA, for $1.5 \mathrm{~h}$ at room temperature in a humidified chamber. After washing twice with PBS, slides were incubated with fluorescein isothiocyanate-conjugated goat anti-mouse IgG (ICN Biomedicals Inc.) diluted $1: 20(50 \mu \mathrm{g} / \mathrm{ml})$ in PBS- $0.1 \%$ BSA for $30 \mathrm{~min}$ at room temperature in a humidified chamber. Following the incubation, slides were washed with PBS three times, airdried, and mounted with Antifade (Molecular Probes, Eugene, OR, USA). Spermatozoa were examined using a fluorescence microscope (Olympus BX40F, Melville, NY, USA). At least 200 cells were counted in different fields and the percentage of spermatozoa showing fluorescence in their tails was calculated. Negative controls were performed by blocking PY20 with ortho-D,L phosphotyrosine (Sigma).

\section{Western blot analysis of sperm proteins}

Proteins from spermatozoa were analyzed by SDS-PAGE and Western immunoblotting. Cells were washed twice with PBS and resuspended in Laemmli sample buffer ( $25 \mathrm{mM}$ Tris, $0.5 \%$ SDS and 5\% glycerol, pH 6.8) (Laemmli 1970). Samples were centrifuged at $6000 \mathrm{~g}$ for $5 \mathrm{~min}$. The supernatants were recovered and heated at $100^{\circ} \mathrm{C}$ for $5 \mathrm{~min}$ in the presence of $70 \mathrm{mM} 2 \beta$-mercaptoethanol and stored at $-20^{\circ} \mathrm{C}$ until use. Solubilized proteins (obtained from $2 \times 10^{6}$ spermatozoa per lane $(\sim 5 \mu \mathrm{g}$ protein)) were separated on $7 \%$ polyacrylamide gels under denaturing conditions. Prestained molecular weight markers (Amersham Life Science Inc., Oakville, Ontario, Canada) were run in parallel. For Western blot analysis, proteins were electroblotted and transferred onto nitrocellulose (BioRad, Hercules, CA, USA) at $100 \mathrm{~V}$ at $4^{\circ} \mathrm{C}$ for $2 \mathrm{~h}$. To block non-specific binding sites, the membrane was first incubated with $2 \%$ dry skimmed milk in PBS$0.1 \%$ Tween 20 (blocking solution). Then it was incubated for $1 \mathrm{~h}$ with the monoclonal anti-phosphotyrosine antibody 4G10 (Upstate Biotechnology, Lake Placid, NY, USA) diluted 1:5000 in blocking solution. After four washes with PBS-0.1\% Tween 20, an anti-mouse peroxidase-conjugated IgG (Jackson Immuno-Research Laboratories Inc., West Grove, PA, USA) diluted 1:5000 in blocking solution was added. Following $1 \mathrm{~h}$ of incubation, the membrane was washed four times with PBS-0.1\% Tween 20, and reactive bands were detected by enhanced chemiluminescence using the ECL kit (Amersham Life Science Inc.) according to the manufacturer's instructions. All incubations were performed at room temperature.

To quantify changes in protein tyrosine phosphorylation, rectangular boxes were drawn around bands on scanned digital images of ECL contact photographs of Western blots, and adjusted optical densities for each lane were obtained using ImageJ software $1.30 \mathrm{~V}$ (National Institutes of Health, Bethesda, MD, USA).

\section{Membrane fluidity}

Sperm membrane fluidity was evaluated using the fluorescent probe 6-dodecanoyl-2-dimethylaminonaphthalene (Laurdan; Molecular Probes Inc., Eugene, OR, USA). Normozoospermic and asthenozoospermic spermatozoa were mixed with Laurdan at 1:1000 (probe:lipid ratio) and incubated for $15 \mathrm{~min}$ at $37^{\circ} \mathrm{C}$ to allow the incorporation of the probe into the membrane. After incubation, membrane fluidity was evaluated by changes in Laurdan generalized polarization (GP) calculated as: GP $=\left(\mathrm{I}_{430}-\mathrm{I}_{480}\right) /\left(\mathrm{I}_{430}+\right.$ $\mathrm{I}_{480}$ ) where $\mathrm{I}_{430}$ and $\mathrm{I}_{480}$ are the fluorescence intensities at $430 \mathrm{~nm}$ and $480 \mathrm{~nm}$ respectively ( $\lambda$ excitation: $350 \mathrm{~nm}$ ) (Harris et al. 2002) measured in a Kontron SFM-25 spectrofluorometer with temperature control (Kontron Instruments SPA, Milan, Italy).

\section{Statistical analysis}

Results are expressed as means \pm S.D. Or S.E.M. Statistical differences between two groups were evaluated by Student's $t$-test. Results obtained from different sperm groups at the same incubation time were compared by two-way ANOVA and Student-Newman-Keuls test. All tests were two-tailed with a statistical significance assessed at the $P<0.05$ level. Statistical analysis was performed using the Graphpad InStat program (GraphPad software, San Diego, CA, USA).

\section{Results}

\section{Motion parameters and hyperactivated motility of spermatozoa incubated under capacitating conditions}

By definition, asthenozoospermic samples showed significantly lower original motility than normozoospermic and fertile samples $(P<0.01$ in both cases) (Table 1). After $6 \mathrm{~h}$ of capacitating incubation, asthenozoospermic samples showed a proportionally greater decrease in motility (31.9\% drop) than the other two groups (normozoospermic, $15.8 \%$ and fertile donors, $13.2 \%$ ). Viability of spermatozoa, on the other hand, was not significantly different among the study groups at any time of incubation.

Judged by motion analysis of the sperm tracks, asthenozoospermic samples also revealed differences in the quality of sperm movement. At T0, they displayed significantly $(P<0.01)$ lower velocity (VAP, VSL, and $\mathrm{VCL})$ compared with normozoospermic and fertile samples. At T6, the difference between normozoospermic and asthenozoospermic samples increased in magnitude and statistical significance $(P<0.001)$. No significant differences were observed between normozoospermic and fertile samples.

Mean values of ALH displacement were significantly $(P<0.05)$ lower for asthenozoospermic when compared with normozoospermic and fertile samples at T0. This 
Table 1 Motion parameters obtained from spermatozoa after 0 (T0) and 6 (T6) h of incubation under capacitating conditions. The results are expressed as means \pm S.D.

\begin{tabular}{|c|c|c|c|c|c|c|}
\hline \multirow[b]{2}{*}{ Variable } & \multicolumn{2}{|c|}{ Normozoospermic } & \multicolumn{2}{|c|}{ Asthenozoospermic } & \multicolumn{2}{|c|}{ Fertile } \\
\hline & T0 & T6 & T0 & T6 & T0 & T6 \\
\hline Motility (\%) & $65.9 \pm 7.7$ & $55.5 \pm 9.2$ & $26.6 \pm 7.0^{\mathrm{a}}$ & $18.1 \pm 5.7^{\mathrm{a}}$ & $64.5 \pm 7.0$ & $56.0 \pm 2.4$ \\
\hline $\operatorname{VAP}(\mu \mathrm{m} / \mathrm{s})$ & $61.0 \pm 9.9$ & $71.8 \pm 10.8$ & $45.4 \pm 7.7^{a}$ & $48.7 \pm 14.9^{\mathrm{a}}$ & $67.7 \pm 9.5$ & $59.5 \pm 16.5$ \\
\hline VSL $(\mu \mathrm{m} / \mathrm{s})$ & $50.6 \pm 8.1$ & $62.5 \pm 9.8$ & $36.0 \pm 6.6^{a}$ & $41.6 \pm 14.4^{\mathrm{a}}$ & $57.7 \pm 10.6$ & $54.0 \pm 17.5$ \\
\hline $\operatorname{VCL}(\mu \mathrm{m} / \mathrm{s})$ & $89.5 \pm 19.8$ & $119.9 \pm 23.0$ & $66.8 \pm 16.8^{\mathrm{a}}$ & $84.3 \pm 13.0^{\mathrm{a}}$ & $102.7 \pm 16.6$ & $82.1 \pm 22.1$ \\
\hline $\mathrm{ALH}(\mu \mathrm{m})$ & $3.9 \pm 0.7$ & $4.9 \pm 1.1$ & $2.9 \pm 0.7^{\mathrm{b}}$ & $3.9 \pm 0.6$ & $4.5 \pm 0.6$ & $4.6 \pm 0.6$ \\
\hline $\mathrm{BCF}(\mathrm{Hz})$ & $25.0 \pm 9.1$ & $26.4 \pm 4.5$ & $23.9 \pm 5.2$ & $33.0 \pm 11.7$ & $23.8 \pm 2.5$ & $25.0 \pm 3.1$ \\
\hline STR (\%) & $75.0 \pm 19.6$ & $85.7 \pm 2.8$ & $80.2 \pm 6.8$ & $75.7 \pm 23.2$ & $80.0 \pm 5.8$ & $87.0 \pm 5.5$ \\
\hline LIN (\%) & $57.9 \pm 5.3$ & $53.6 \pm 7.1$ & $55.1 \pm 7.8$ & $50.8 \pm 9.6$ & $53.8 \pm 4.4$ & $57.0 \pm 3.3$ \\
\hline Viability (\%) & $93.6 \pm 3.0$ & $89.4 \pm 2.7$ & $89.9 \pm 5.3$ & $80.5 \pm 7.8$ & $95.0 \pm 0.5$ & $90.3 \pm 3.3$ \\
\hline
\end{tabular}

Superscripts a and b represent statistical differences between asthenozoospermic and normozoospermic samples at the same incubation time $(P<0.01$ and $P<0.05$ respectively). No significant differences $(P>0.05)$ were observed between normozoospemeric and proven-fertile samples.

difference was no longer statistically significant at T6. Furthermore, no statistical differences were observed for values of flagellar BCF, STR, and LIN of trajectory at any incubation time.

The studied groups revealed different abilities to develop hyperactivated motility when they were incubated under capacitating conditions for $6 \mathrm{~h}$ (Fig. 1A). Out of the total number of motile spermatozoa, the percentage of hyperactivated cells in asthenozoospermic samples was
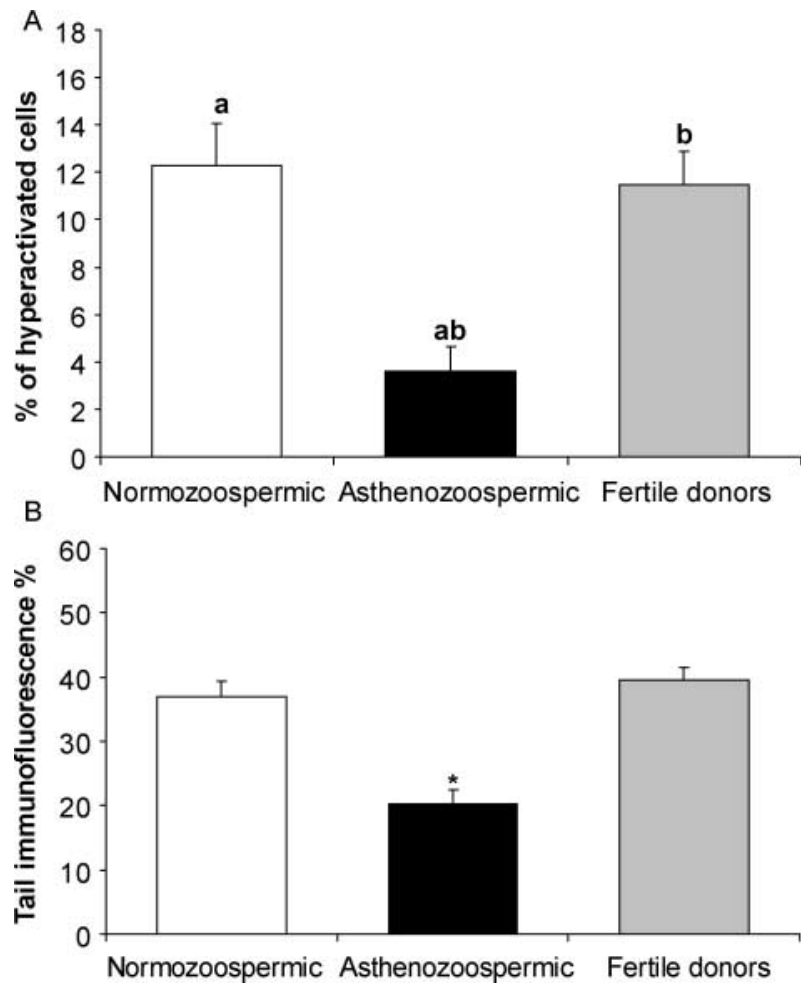

Figure 1 Percentage of (A) hyperactivated spermatozoa and (B) phosphotyrosine $(\mathrm{pY})$ immunoreactive sperm after $6 \mathrm{~h}$ of incubation under capacitating conditions. Results are means \pm S.E.M. Values with the same superscript differ: $\mathrm{a}, P<0.01$ and $\mathrm{b}, P<0.05 . * P<0.05$ in comparison with normozoospermic and fertile donors. significantly lower than that observed in normozoospermic $(P<0.01)$ and fertile samples $(P<0.05)$.

\section{Protein tyrosine phosphorylation under conditions conducive to capacitation}

The incidence of tyrosine phosphorylation in the described sperm populations was determined by immunofluorescence with a specific anti-phosphotyrosine antibody, while the intensity of tyrosine phosphorylation was evaluated on Western blots of total sperm protein extracts. Although spermatozoa showed phosphotyrosine immunolabeling on both head and tail, the signal associated with tail proteins was stronger and more consistent than that of the head; tail labeling was therefore used to consider a spermatozoon as 'positive' in the immunofluorescence assessment (Fig. 2).

After $6 \mathrm{~h}$ of incubation under capacitating conditions, the incidence of spermatozoa with phosphotyrosineimmunoreactive tails (Fig. 1B) was significantly lower in the asthenozoospermic group than in the normozoospermic and fertile donor groups $(P<0.05)$. These latter groups were not statistically different from one another. The difference between asthenozoospermic and normozoospermic samples was observed regardless of the method used to separate sperm from seminal plasma (data not shown).

Similar results were observed following the Western blot analysis of spermatozoa (Fig. 3). Major differences were observed in the level of tyrosine-phosphorylated proteins recovered from the three groups studied. The lowest signal was detected in proteins extracted from spermatozoa of asthenozoospermic patients (Fig. 3). Densitometric analysis of the major protein bands (range: $77-170 \mathrm{kDa}$ ) showed that at T6 normozoospermic samples revealed a 1.8-fold increase in the overall signal compared with T0. The average increase in signal for fertile samples was 1.9-fold. Asthenozoospermic samples, however, showed no increase in the signal intensity of most of their protein bands at T6, revealing an inability to respond to capacitating conditions with the enhanced tyrosine phosphorylation observed in normozoospermic and fertile samples. 


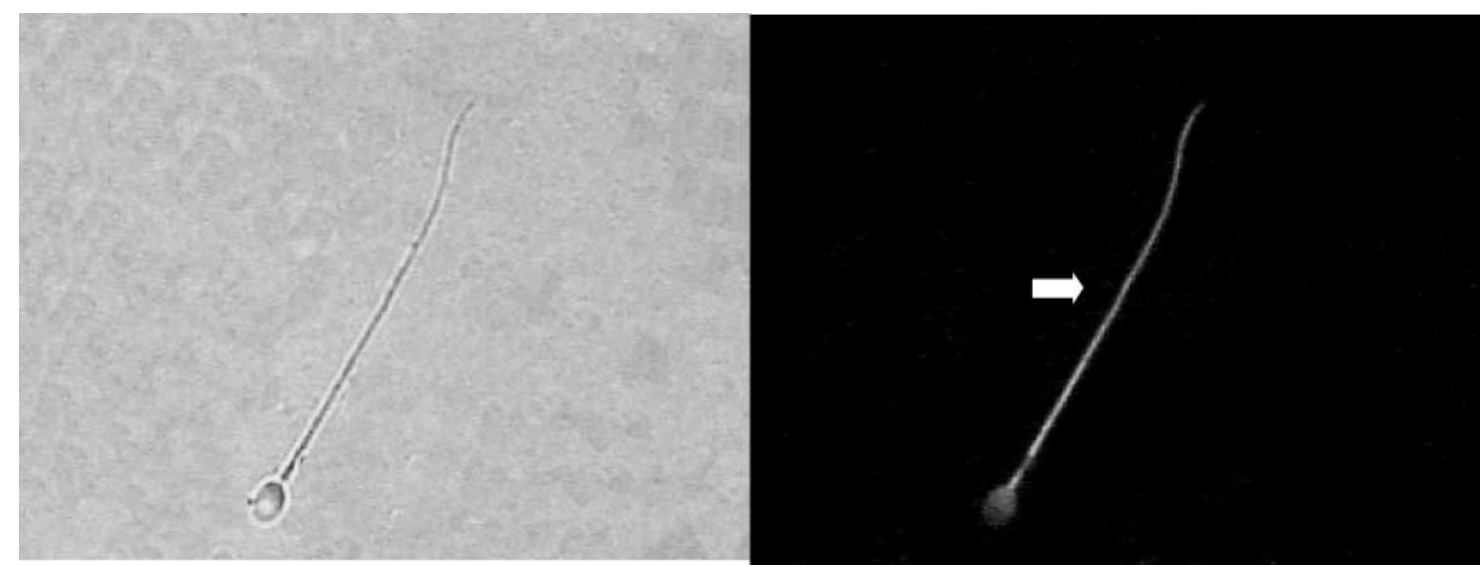

Figure 2 Immunofluorescent localization of phosphotyrosine proteins on human spermatozoa incubated under capacitating conditions as detected by immunoreactivity with the anti-phosphotyrosine monoclonal antibody PY20. (Left) Phase contrast and (right) corresponding immunofluorescence photographs. The arrow shows the immunolocalization of phosphotyrosine along the principal piece of the sperm tail.

\section{Effect of cAMP analogs and pentoxifylline on protein tyrosine phosphorylation}

To evaluate whether spermatozoa from asthenozoospermic samples could overcome their tyrosine phosphorylation deficiency when the kinase system was directly stimulated bypassing the plasma membrane, we designed an experiment where sperm were incubated with PTX and dbcAMP. Spermatozoa from the normozoospermic and asthenozoospermic groups were incubated for $6 \mathrm{~h}$ in capacitating conditions, adding PTX $(1 \mathrm{mM})$ and dbcAMP $(1 \mathrm{mM})$ during the last hour to increase their endogenous levels of CAMP and induce tyrosine phosphorylation.

Baseline incidence of phosphotyrosine-immunoreactive spermatozoa after a capacitating incubation (T6) was significantly lower $(P<0.05)$ in the asthenozoospermic than in the normozoospermic group (Fig. 4). cAMP stimulation induced a statistically significant $(P<0.05)$ increase in the number of tyrosine-phosphorylated spermatozoa in the asthenozoospermic group, which was equal to or even greater in relative magnitude to that of spermatozoa from normozoospermic samples, suggesting that the main block to increasing tyrosine phosphorylation during capacitation resides upstream of the CAMPdependent kinase(s).

\section{Assessment of membrane fluidity}

Since sperm membrane changes have been associated with capacitation-triggered tyrosine phosphorylation and precede cAMP production, we decided to assess sperm membrane fluidity in normo- and asthenozoospermic spermatozoa. Sperm membrane fluidity was evaluated following the variation in the GP of the fluorescent probe Laurdan (Fig. 5). This molecule spontaneously incorporates into membranes at the glycerol backbone level, distributing itself evenly among the different lipid domains (Parasassi \& Gratton 1995). Laurdan responds to variations in the number of water molecules accessible to the probe, which in turn depends on both lipid packing and cholesterol content. This alteration in the polarity of Laurdan's microenvironment is visualized as an alteration in its fluorescence excitation and emission spectra. Results are expressed as ratios of the probe's polarization. The lower the ratio, the higher the fluidity of the membrane. Asthenozoospermic samples showed a $21 \%$ higher GP ratio $(P<0.0001)$ prior to their capacitation $(\mathrm{T} 0)$ in comparison with normozoospermic samples $(0.097 \pm 0.002$ and $0.117 \pm 0.004$ for normozoospermic and asthenozoospermic spermatozoa respectively), indicating lower membrane fluidity.

When incubated for $6 \mathrm{~h}$ in a capacitating medium, both normozoospermic and asthenozoospermic cells significantly decreased their GP values $(P<0.01)$ in comparison with those found before capacitation, reflecting an increase

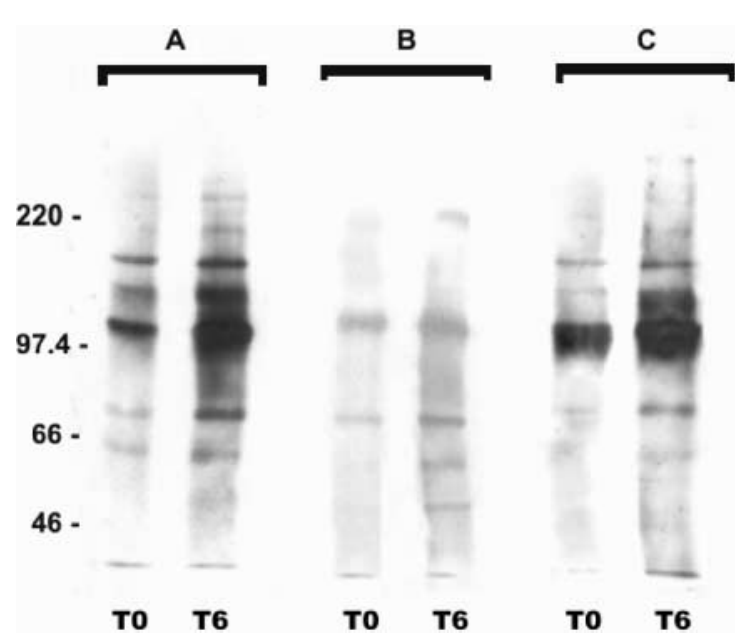

Figure 3 Western blot analysis of sperm tyrosine-phosphorylated proteins extracted immediately after washing (T0) and after $6 \mathrm{~h}$ (T6) of incubation under capacitating conditions. Representative blot from 24 experiments. A, normozoospermic; B, asthenozoospermic; C, fertile. 


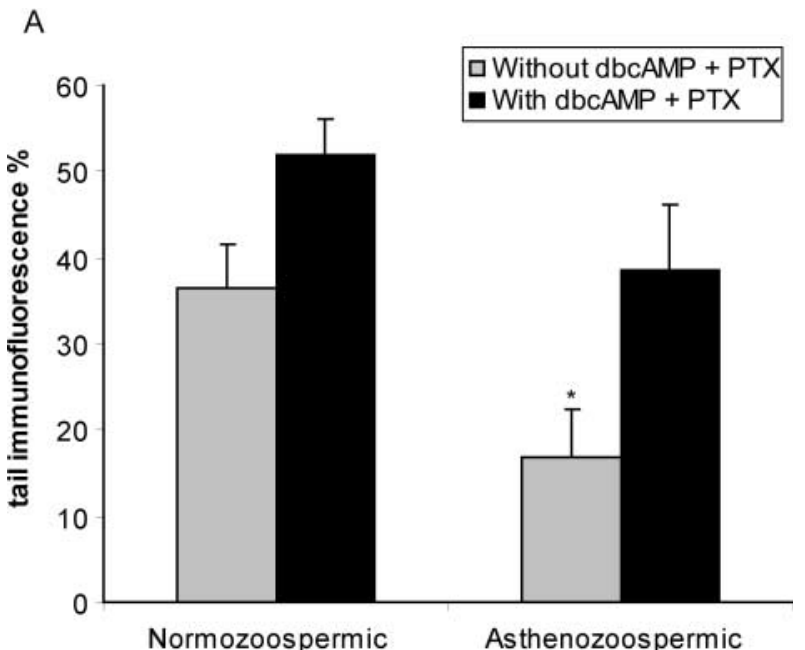

B

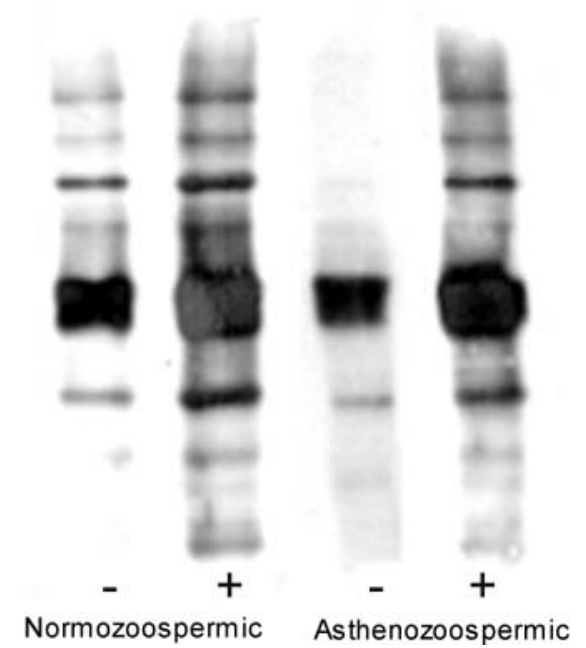

Figure 4 Percentage of phosphotyrosine $(\mathrm{pY})$ immunoreactive sperm (upper, immunofluorescence) and intensity of pY proteins (lower, Western blot) in normozoospermic and asthenozoospermic samples after $6 \mathrm{~h}$ of incubation under capacitating conditions with $(+)$ or without (-) PTX and dbcAMP. Results are expressed as means \pm S.E.M. The experiments showed an increase in the incidence of phosphotyrosine-immunoreactive spermatozoa, as well as in the intensity of $\mathrm{pY}$ phosphorylation, in normozoospermic and asthenozoospermic samples. Spermatozoa from asthenozoospermic samples incubated with PTX and dbcAMP achieved a percentage of tyrosine-phosphorylated cells and a level of pY phosphorylation similar to that of untreated spermatozoa from normozoospermic samples, overcoming their original deficiency. $*$ Statistically different from sperm incubated without PTX and dbcAMP $(P<0.05)(n=4)$.

in membrane fluidity. However, even when the magnitude of the changes was similar for both groups, the overall fluidity of asthenozoospermic cells remained significantly lower $(P<0.05)$ than that attained by spermatozoa from the normozoospermic group after capacitation. These differences were observed irrespective of the method used to separate sperm from seminal plasma (data not shown).

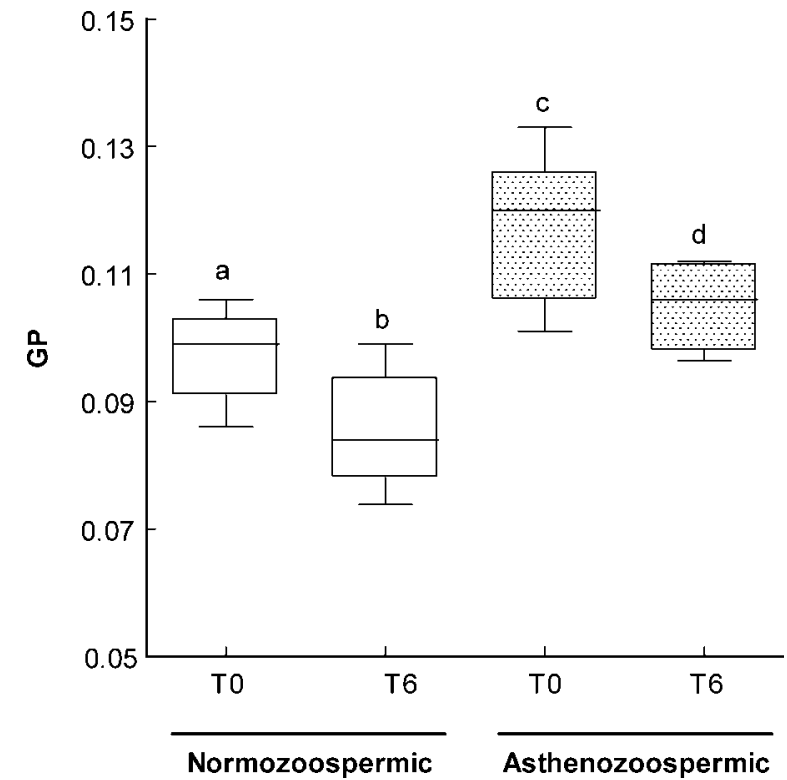

Figure 5 Membrane fluidity of spermatozoa studied immediately after washing (T0) and after $6 \mathrm{~h}$ (T6) of incubation under capacitating conditions and measured by the variation of the Laurdan GP. Results are expressed as median \pm S.E.M. Statistically significant: $a$ vs $b, P<0.01$; c vs d, $P<0.05 ;$ a vs $\mathrm{c}, P<0.01 ; \mathrm{b}$ vs $\mathrm{d}, P<0.05$.

\section{Discussion}

Asthenozoospermia is a common cause of sperm pathology and male infertility (Aitken et al. 1982). Except for those cases of severe alteration in which ultrastructural flagellar anomalies are detected (Chemes et al. 1998), the etiology of this sperm pathology likely involves an array of biochemical and functional defects. In our study, spermatozoa from asthenozoospermic patients showed a clear impairment in their motility and their capacity to develop hyperactivation, which associated with low membrane fluidity and a concomitant inability to undergo protein tyrosine phosphorylation. This was particularly evident when spermatozoa were challenged with a capacitating incubation $\left(6 \mathrm{~h}\right.$ at $37^{\circ} \mathrm{C}, 5 \% \mathrm{CO}_{2}$ in Ham/BSA).

Semen samples from asthenozoospermic patients showed not only a low percentage of motile spermatozoa but sperm motion of poor quality, reflected by decreased VAP, VSL, VCL, and ALH. As mentioned, these spermatozoa also displayed a marked impairment in their capacity to develop hyperactivated motility. Although these defects may be the cause of the reported poor fertilizing ability of asthenozoospermic samples, the pathogenetic mechanisms leading to such alterations are, for the most part, unknown.

Several groups, including ours, have reported a close association between sperm protein tyrosine phosphorylation and motility and hyperactivation in different mammalian species (Leclerc et al. 1996, Nassar et al. 1999, Si \& Okuno 1999, Luconi et al. 2001, Yunes et al. 2003, Buffone et al. 2004). Differences in the level of hyperactivated sperm, especially after capacitation, 
could originate from the impaired capacity of these cells to phosphorylate proteins in tyrosine residues which, in turn, could be linked to reduced membrane fluidity.

Various reports have described an active participation of the sperm plasma membrane in the process of capacitation, mainly through loss of cholesterol (Davis 1981, Cross 1998, Visconti et al. 1999a). Cholesterol efflux produces an increase in membrane fluidity which directly or indirectly impacts protein function, such as that of surface ion channels and enzymes. Changes in ion fluxes across the membrane, mainly $\mathrm{HCO}_{3}^{-}, \mathrm{Na}^{+}, \mathrm{K}^{+}$, and $\mathrm{Ca}^{2+}$, have been implicated in initiating sperm capacitation as well as tyrosine phosphorylation (Yanagimachi 1994, Visconti \& Kopf 1998). More specifically, an increase in intracellular $\mathrm{HCO}_{3}^{-}$stimulates soluble adenyl cyclase which rapidly increases CAMP, protein tyrosine phosphorylation, sperm motility, and acrosome reaction (Visconti et al. 1999b, Gadella \& Harrison 2000, Luconi et al. 2005). The endpoint of these capacitation-associated changes, at least those related to motility and hyperactivation, appears to be an increased binding of the regulatory subunits of PKA, especially typell, to tyrosine-phosphorylated forms of A-kinase anchoring proteins 3 and 4 . Interruption of this binding leads to decreased sperm motility (Vijayaraghavan et al. 1997, Luconi et al. 2004).

Sperm tyrosine phosphorylation can be stimulated by cAMP analogs and/or phosphodiesterase inhibitors via direct PKA activation, which occurs downstream of the plasma membrane (Visconti et al. 1995b, Leclerc et al. 1996, Nassar et al. 1999, Bajpai \& Doncel 2003, Buffone et al. 2004). In order to evaluate the participation of a plasma membrane defect in the inability of asthenozoospermic sperm to undergo protein tyrosine phosphorylation, we designed an experiment in which spermatozoa were treated with dbcAMP and pentoxyfilline to increase their endogenous levels of cAMP. Results showed that defective tyrosine phosphorylation of asthenozoospermic spermatozoa could be overcome when those stimulators were added to the incubation medium, indicating that signal transduction mechanisms downstream of cAMP were not significantly affected in those cells.

These findings suggested that the deficiency in tyrosine phosphorylation observed in asthenozoospermic spermatozoa and, possibly, their dysfunctional motility could be associated with an alteration in the dynamics of the plasma membrane. Removal of cholesterol and increased membrane fluidity is a key step in the initiation of capacitation (Cross 1998). It decreases the cholesterol/phospholipid ratio (Davis 1981), allowing for redistribution of membrane lipids and proteins (Cross \& Overstreet 1987, Gadella et al. 1999). Capacitation can be inhibited by adding cholesterol or cholesterol analogs to the capacitating medium (Visconti et al. 1999a), and can be stimulated by cholesterol acceptors such as $\beta$-cyclodextrins (Osheroff et al. 1999). Changes in membrane dynamics have been associated with tyrosine phosphorylation as well as sperm function and fertilizing ability (Gadella et al. 1999, Flesch et al. 2001).
In order to verify if asthenozoospermic sperm have a membrane dynamics defect that could be associated with their tyrosine phosphorylation and motility deficiencies, we have measured changes in the membrane fluidity of the sperm during a capacitating incubation, studying changes in Laurdan fluorescence (Ambrosini et al. 2001). Spermatozoa from the asthenozoospermic group revealed significantly less fluid membranes at the beginning of the incubation (T0). Capacitation increased the membrane fluidity of these cells, but never to the level achieved by normozoospermic spermatozoa.

Such difference in membrane fluidity could be due to the increased susceptibility of these spermatozoa to suffer peroxidative damage (Calamera et al. 2003), as the generation of membrane lipid hydroperoxides has been associated with membrane fluidity reduction (Aitken et al. 1993, 1994, Windsor et al. 1993). This susceptibility of asthenozoospermic sperm could be explained, in part, by their membrane composition, which is responsible for their reported higher oxidation coefficient (Calamera et al. 2003). Sperm membranes of asthenozoospermic samples contain high levels of polyunsaturated fatty acids, making them more prone to attack by reactive oxygen species. Since oxidizing conditions are normal during sperm capacitation and have been linked to signal transduction and tyrosine phosphorylation (Aitken et al. 1994, 1998), the predisposition of the asthenozoospermic samples to oxidative damage may be the origin of their membrane dysfunction, resulting in tyrosine phosphorylation deficiency and alteration of motility.

The biological and clinical significance of the abovedescribed findings resides in the association between a common sperm pathology such as asthenozoospermia and a defective molecular mechanism, closely related with the acquisition of the sperm's fertilizing ability. This association represents a step toward the elucidation of defective molecular mechanisms that may be the real cause of the reduced fertilizing capacity of pathological sperm.

\section{Acknowledgements}

The authors wish to thank CONRAD (US Agency for International Development; USAID) for supporting G F D. The views of the authors do not necessarily reflect those of CONRAD or USAID. The authors also wish to thank Ms Christine J Farrigan for her excellent editorial assistance. The authors declare that there is no conflict of interest that would prejudice the impartiality of this scientific work.

\section{References}

Aitken RJ, Best FS, Richardson DW, Djahanbakhch O, Mortimer D, Templeton AA \& Lees MM 1982 An analysis of sperm function in cases of unexplained infertility: conventional criteria, movement characteristics, and fertilizing capacity. Fertility and Sterility $\mathbf{3 8}$ $212-221$.

Aitken RJ, Harkiss D \& Buckingham DW 1993 Analysis of lipid peroxidation mechanisms in human spermatozoa. Molecular Reproduction and Development 35 302-315. 
Aitken J, Krausz C \& Buckingham D 1994 Relationships between biochemical markers for residual sperm cytoplasm, reactive oxygen species generation, and the presence of leukocytes and precursor germ cells in human sperm suspensions. Molecular Reproduction and Development 39 268-279.

Aitken RJ, Harkiss D, Knox W, Paterson M \& Irvine DS 1998 A novel signal transduction cascade in capacitating human spermatozoa characterised by a redox-regulated, cAMP-mediated induction of tyrosine phosphorylation. Journal of Cell Science $\mathbf{1 1 1}$ 645-656.

Ambrosini A, Zolese G, Balercia G, Bertoli E, Arnaldi G \& Mantero F 2001 Laurdan fluorescence: a simple method to evaluate sperm plasma membrane alterations. Fertility and Sterility 76 501-505.

Bajpai M \& Doncel GF 2003 Involvement of tyrosine kinase and cAMP-dependent kinase cross-talk in the regulation of human sperm motility. Reproduction 126 183-195.

Bajpai M, Asin S \& Doncel GF 2003 Effect of tyrosine kinase inhibitors on tyrosine phosphorylation and motility parameters in human sperm. Archives of Andrology 49 229-246.

Buffone MG, Doncel GF, Marin Briggiler CI, Vazquez-Levin MH \& Calamera JC 2004 Human sperm subpopulations: relationship between functional quality and protein tyrosine phosphorylation. Human Reproduction 19 139-146.

Burkman LJ 1984 Characterization of hyperactivated motility by human spermatozoa during capacitation: comparison of fertile and oligozoospermic sperm populations. Archives of Andrology 13 153-165.

Calamera J, Buffone M, Ollero M, Alvarez J \& Doncel GF 2003 Superoxide dismutase content and fatty acid composition in subsets of human spermatozoa from normozoospermic, asthenozoospermic, and polyzoospermic semen samples. Molecular Reproduction and Development 66 422-430.

Carrera A, Moos J, Ning XP, Gerton GL, Tesarik J, Kopf GS \& Moss SB 1996 Regulation of protein tyrosine phosphorylation in human sperm by a calcium/calmodulin-dependent mechanism: identification of A kinase anchor proteins as major substrates for tyrosine phosphorylation. Developmental Biology $\mathbf{1 8 0}$ 284-296.

Chemes HE, Olmedo SB, Carrere C, Oses R, Carizza C, Leisner M \& Blaquier J 1998 Ultrastructural pathology of the sperm flagellum: association between flagellar pathology and fertility prognosis in severely asthenozoospermic men. Human Reproduction $\mathbf{1 3}$ 2521-2526.

Cross NL 1998 Role of cholesterol in sperm capacitation. Biology of Reproduction 59 7-11.

Cross NL \& Overstreet JW 1987 Glycoconjugates of the human sperm surface: distribution and alterations that accompany capacitation in vitro. Gamete Research 16 23-35.

Davis BK 1981 Timing of fertilization in mammals: sperm cholester$\mathrm{ol} /$ phospholipid ratio as a determinant of the capacitation interval. PNAS 78 7560-7564.

Flesch FM, Wijnand E, van de Lest $\mathrm{CH}$, Colenbrander B, van Golde LM \& Gadella BM 2001 Capacitation dependent activation of tyrosine phosphorylation generates two sperm head plasma membrane proteins with high primary binding affinity for the zona pellucida. Molecular Reproduction and Development 60 107-115.

Gadella BM \& Harrison RA 2000 The capacitating agent bicarbonate induces protein kinase A-dependent changes in phospholipid transbilayer behavior in the sperm plasma membrane. Development 127 2407-2420.

Gadella BM, Flesch FM, van Golde LM \& Colenbrander B 1999 Dynamics in the membrane organization of the mammalian sperm cell and functionality in fertilization. Veterinary Quarterly 21 $142-146$.

Harris FM, Best KB \& Bell JD 2002 Use of laurdan fluorescence intensity and polarization to distinguish between changes in membrane fluidity and phospholipid order. Biochimica et Biophysica Acta 1565 123-128.

Kruger TF, Menkveld R, Stander FS, Lombard CJ, Van der Merwe JP, van Zyl JA \& Smith K 1986 Sperm morphologic features as a prognostic factor in in vitro fertilization. Fertility and Sterility $\mathbf{4 6}$ 1118-1123.

Laemmli UK 1970 Cleavage of structural proteins during the assembly of the head of bacteriophage T4. Nature 227 680-685.

Leclerc P, de Lamirande E \& Gagnon C 1996 Cyclic adenosine 3',5'monophosphate-dependent regulation of protein tyrosine phosphorylation in relation to human sperm capacitation and motility. Biology of Reproduction 55 684-692.

Luconi M, Marra F, Gandini L, Filimberti E, Lenzi A, Forti G \& Baldi E 2001 Phosphatidylinositol 3-kinase inhibition enhances human sperm motility. Human Reproduction 16 1931-1937.

Luconi M, Carloni V, Marra F, Ferruzzi P, Forti G \& Baldi E 2004 Increased phosphorylation of AKAP by inhibition of phosphatidylinositol 3-kinase enhances human sperm motility through tail recruitment of protein kinase A. Journal of Cell Science 117 $1235-1246$.

Luconi M, Porazzi I, Ferruzzi P, Marchiani S, Forti G \& Baldi E 2005 Tyrosine phosphorylation of the A kinase anchoring protein 3 (AKAP3) and soluble adenylate cyclase are involved in the increase of human sperm motility by bicarbonate. Biology of Reproduction 72 22-32.

Mortimer ST, Swan MA \& Mortimer D 1998 Effect of seminal plasma on capacitation and hyperactivation in human spermatozoa. Human Reproduction 13 2139-2146.

Nassar A, Mahony M, Morshedi M, Lin MH, Srisombut C \& Oehninger S 1999 Modulation of sperm tail protein tyrosine phosphorylation by pentoxifylline and its correlation with hyperactivated motility. Fertility and Sterility 71 919-923.

Osheroff JE, Visconti PE, Valenzuela JP, Travis AJ, Alvarez J \& Kopf GS 1999 Regulation of human sperm capacitation by a cholesterol efflux-stimulated signal transduction pathway leading to protein kinase A-mediated up-regulation of protein tyrosine phosphorylation. Molecular Human Reproduction 5 1017-1026.

Parasassi T \& Gratton E 1995 Membrane lipid domains and dynamics as detected by Laurdan fluorescence. Journal of Fluorescence $\mathbf{5}$ $59-69$.

Pukazhenthi BS, Long JA, Wildt DE, Ottinger MA, Armstrong DL \& Howard J 1998 Regulation of sperm function by protein tyrosine phosphorylation in diverse wild felid species. Journal of Andrology 19 675-685.

Sakkas D, Leppens-Luisier G, Lucas H, Chardonnens D, Campana A, Franken DR \& Urner F 2003 Localization of tyrosine phosphorylated proteins in human sperm and relation to capacitation and zona pellucida binding. Biology of Reproduction $\mathbf{6 8}$ $1463-1469$.

Si Y \& Okuno M 1999 Role of tyrosine phosphorylation of flagellar proteins in hamster sperm hyperactivation. Biology of Reproduction 61 240-246.

Vijayaraghavan S, Goueli SA, Davey MP \& Carr DW 1997 Protein kinase A-anchoring inhibitor peptides arrest mammalian sperm motility. Journal of Biological Chemistry 272 4747-4752.

Visconti PE \& Kopf GS 1998 Regulation of protein phosphorylation during sperm capacitation. Biology of Reproduction 59 1-6.

Visconti PE, Bailey JL, Moore GD, Pan D, Olds-Clarke P \& Kopf GS 1995a Capacitation of mouse spermatozoa. I. Correlation between the capacitation state and protein tyrosine phosphorylation. Development 121 1129-1137.

Visconti PE, Moore GD, Bailey JL, Leclerc P, Connors SA, Pan D, Olds-Clarke P \& Kopf GS 1995b Capacitation of mouse spermatozoa. II. Protein tyrosine phosphorylation and capacitation are regulated by a CAMP-dependent pathway. Development 121 1139-1150.

Visconti PE, Ning X, Fornes MW, Alvarez JG, Stein P, Connors SA \& Kopf GS 1999a Cholesterol efflux-mediated signal transduction in mammalian sperm: cholesterol release signals an increase in protein tyrosine phosphorylation during mouse sperm capacitation. Developmental Biology 214 429-443.

Visconti PE, Stewart-Savage J, Blasco A, Battaglia L, Miranda P, Kopf GS \& Tezon JG 1999b Roles of bicarbonate, cAMP, and 
protein tyrosine phosphorylation on capacitation and the spontaneous acrosome reaction of hamster sperm. Biology of Reproduction 61 76-84.

Visconti PE, Westbrook VA, Chertihin O, Demarco I, Sleight S \& Diekman AB 2002 Novel signaling pathways involved in sperm acquisition of fertilizing capacity. Journal of Reproductive Immunology 53 133-150.

Windsor DP, White IG, Selley ML \& Swan MA 1993 Effects of the lipid peroxidation product (E)-4-hydroxy-2-nonenal on ram sperm function. Journal of Reproduction and Fertility 99 359-366.

World Health Organization (WHO) 1999 In Laboratory Manual for the Examination of Human Semen and Sperm-Cervical Mucus Interaction. Cambridge: Cambridge University Press.

Yanagimachi R 1994 Mammalian fertilization. In The Physiology of Reproduction, edn 2, pp 189-317. Ed. E Knobil. New York: Raven Press.
Yeung CH, Weinbauer GF \& Cooper TG 1999 Responses of monkey epididymal sperm of different maturational status to second messengers mediating protein tyrosine phosphorylation, acrosome reaction, and motility. Molecular Reproduction and Development 54 194-202.

Yunes R, Doncel GF \& Acosta AA 2003 Incidence of sperm-tail tyrosine phosphorylation and hyperactivated motility in normozoospermic and asthenozoospermic human sperm samples. Biocell 27 29-36.

Received 28 November 2004

First decision 14 January 2005

Revised manuscript received 21 February 2005

Accepted 1 March 2005 